\title{
Coexistence of Spinal Intramedullary Tuberculoma and Multiple Intracranial Tuberculomas
}

\author{
Dong-Yoon Lee ${ }^{1}$, Sang-Pyo $\mathrm{Kim}^{2}$, In-Soo Kim ${ }^{1}$ \\ Departments of ${ }^{I}$ Neurosurgery, ${ }^{2}$ Pathology, Dongsan Medical Center, Keimyung University School of Medicine, Daegu, Korea
}

Spinal intramedullary tuberculoma remains a very rare entity of central nervous system tuberculosis. This is the same with the coexistence of spinal intramedullary and intracranial tuberculomas that remains extremely rare with less than 20 cases reported at present. Authors describe this uncommon case by analyzing a 65-year-old female patient who had past history of kidney transplantation due to stage 5 chronic kidney disease and pulmonary tuberculosis on medication. The patient experiences progressive paraplegia and numbness on both lower extremities. Magnetic resonance imaging demonstrated an intramedullary mass at T9-10 level and multiple intracranial enhancing nodules. Microsurgical resection of spinal intramedullary mass was performed and the lesion was histopathologically confirmed as Mycobacterium tuberculosis. Efficient diagnosis and management of this rare disease are reviewed along with previously reported cases.

Key Words: Intramedullary · Intracranial · Tuberculoma

\section{INTRODUCTION}

Tuberculosis (TB) is an infectious bacterial disease caused by Mycobacterium tuberculosis that most commonly affects the lungs. It is transmitted from one person to another through the droplets from the throat and the lungs of people with active respiratory disease. Mostly because of the increasing prevalence of HIV, there has been a recent resurgence of TB in developing and developed countries. Central nervous system (CNS) tuberculoma is unusual compared with the other systems in the body. After the analysis of the involvement of CNS analyzed in $0.5-2 \%$ of the total TB patients, it generally presents an intracranial lesion, while spinal intramedullary tuberculoma is rarely seen at a rate of 2/1,000 cases of CNS tuberculomas ${ }^{18)}$. The coexistence of intracranial and intramedullary tuberculoma is also extremely rare. Because of this, the authors reported this uncommon case of CNS TB that occurred in a 65-year-old immunocompromised female because of kidney transplantation and pulmonary TB.

- Received: February 3, 2015 • Revised: May 12, 2015

- Accepted: May 20, 2015

Corresponding Author: In-Soo Kim, MD, PhD

Department of Neurosurgery, Dongsan Medical Center, Keimyung

University, School of Medicine, Dalseong-Ro, Jung-Gu, Daegu

700-712, Korea

Tel: +82-53-250-7730, Fax: +82-53-250-7356

E-mail: neurokim@dsmc.or.kr

$\otimes$ This is an Open Access article distributed under the terms of the Creative

Commons Attribution Non-Commercial License (http://creativecommons.org/

licenses/by-nc/3.0/) which permits unrestricted non-commercial use, distribution,

and reproduction in any medium, provided the original work is properly cited.

\section{CASE REPORT}

A 65-year-old female visited the emergency department, complaining about headache, paraplegia, numbness of both legs, and urinary incontinence for one week. She had a history of kidney transplantation because of a stage 5 chronic kidney disease. She was also diagnosed with active pulmonary TB two weeks ago and started taking the combination of antituberculosis medication (isoniazid, ethambutol, rifabutin, and pyridoxine). Neurologic examination revealed only a trace or a flicker of movement on both lower extremities and impaired sensations below T10 level, while the motor and sensory functions of upper extremities were normal. The anal sphincter tone was decreased, and the deep tendon reflexes were absent on both lower limbs.

The spine magnetic resonance imaging (MRI) showed a round, well-defined, intramedullary-enhancing lesion $(7 \times 6 \times$ $14 \mathrm{~mm}$ in size) at T9-T10 levels with high signal change of spinal cord at T5-L1 levels (Fig. 1). Surgical resection was performed to decompress the spinal cord. There was a noted greyish, well-demarcated, firm mass that was radically removed. Consistent with tuberculosis, the histopathologic examination showed noncaseating, granulomatous inflammation. A few acid-fast bacilli were detected in granuloma (Fig. 2), and the neurologic deficit was not fully recovered right after the resection. After surgery, patient complains of persistent headache, so we took brain MRI in order to differentiate the brain lesion and it revealed multiple rim-enhancing nodular lesions which 


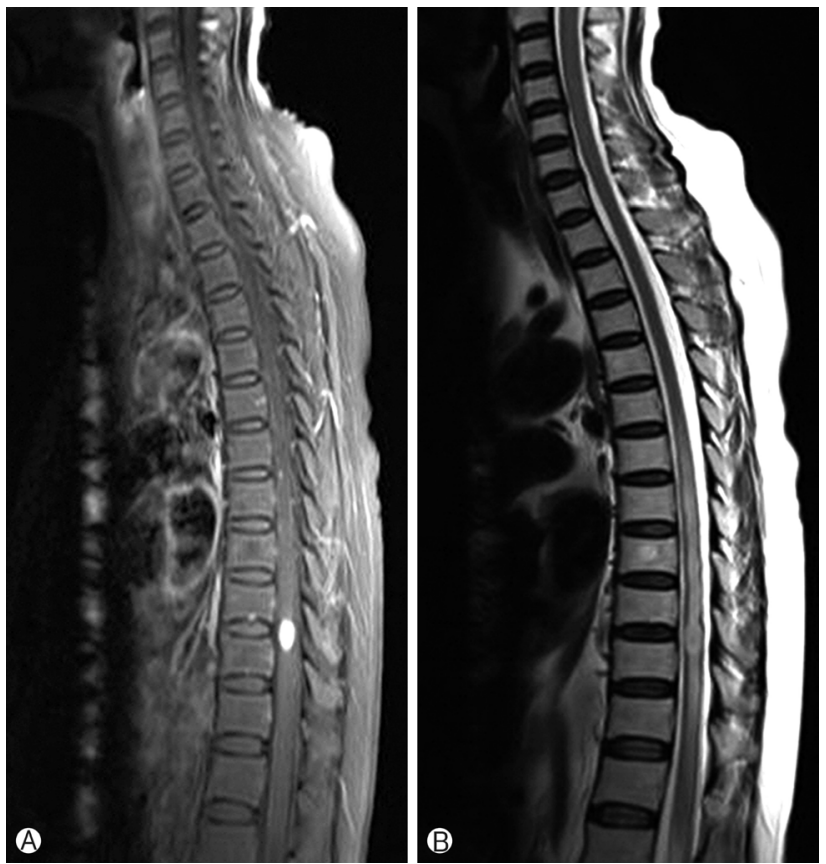

Fig. 1. Sagittal spinal magnetic resonance imaging revealed a round shaped, well enhanced, intramedullary mass lesion on T9T10 level on contrast enhanced $\mathrm{T} 1$-weighted image (A) with hyperintense cord edema expanding from T6 to T12 on T2-weighted sagittal image $(\mathrm{B})$.

were suggestive of tuberculomas (Fig. 3). Anti-tuberculosis medications were persisted with rehabilitation program.

\section{DISCUSSION}

In patients afflicted with human immunodeficiency virus (HIV) infection, as well as transplant recipients, tuberculosis is among the most crucial and opportunistic infections. With this, the aforementioned patient populations are at a high relative risk of acquiring disseminated TB. Such risk is further complicated as these patients receive medications that make treatment difficult ${ }^{12)}$. Another patient population with a heightened TB acquisition risk is those who underwent solid organ transplant (SOT) that was followed by immunosuppression. Moreover, these patients are at an increased risk for extrapulmonary TB. SOT patients are 20-74 times more likely to acquire TB in comparison with the general population ${ }^{8,15)}$. For transplant recipients, a majority of those that are infected with $\mathrm{TB}$ is because of the reactivation of dormant diseases. However, there have also been reported cases wherein nosocomial infection and donor transmission were the causes ${ }^{10}$. SOT patients who become afflicted with CNS tuberculosis after their transplant may have the disease for up to 11 years ${ }^{5,19)}$. Also, while it is assumed that immunosuppression and como-

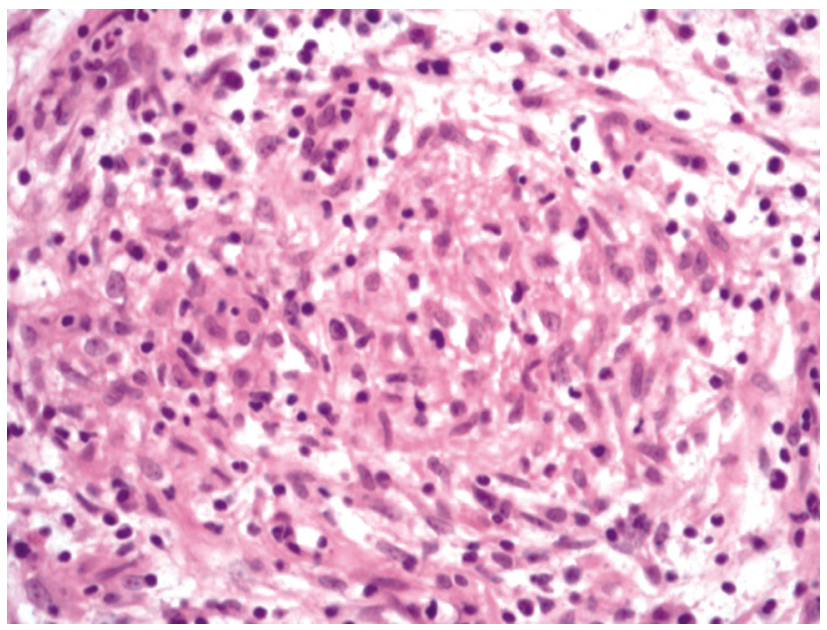

Fig. 2. Histopathologic examination shows noncaseating granulomas with Langhans type giant cells, epithelioid histiocytes, and lymphocytes (hematoxylin and eosin, original magnification $\times 400$ ).

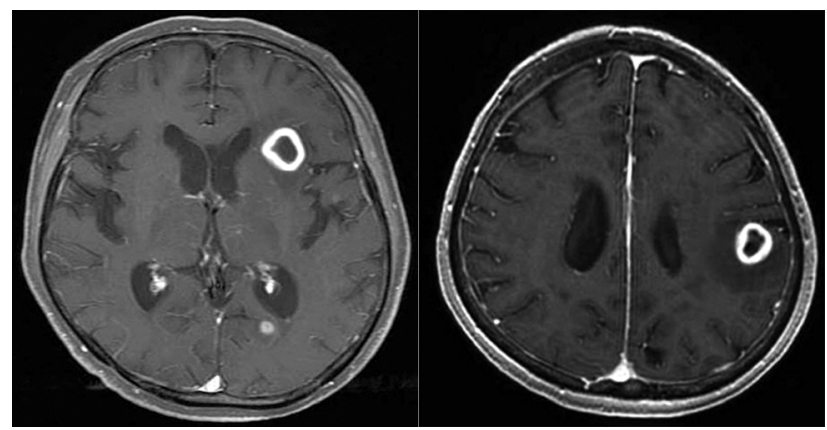

Fig. 3. T1-weighted axial brain MRI demonstrated multiple, rim-enhancing lesion on left frontal lobe and parietal lobe, which was suggestive of tuberculoma in the brain.

rbidities may further complicate the presentation of posttransplantation CNS tuberculosis, there is not enough number of reported cases to allow comment on potential differences in clinical presentation ${ }^{12}$.

CNS tuberculomas remains rare, especially in terms of spinal intramedullary tuberculomas. Spinal intramedullary tuberculoma occurs much less frequent than intracranial tuberculoma (with a ratio of 1:42), and it is most commonly located in the thoracic cord $\mathrm{d}^{1,2,4,9,11,16)}$. The higher incidence that involves the dorsolumbar area is explained based on the regional blood flow to the spinal cord and the thoracic cord that receives about $45 \%$ of the entire supply to the cord against $34 \%$ for the cervical cord ${ }^{7,1720)}$. Intramedullary tuberculomas are almost secondary to hematogenous dissemination of TB from elsewhere in the body, usually from the lung ${ }^{13,14}$. In the present case, CNS tuberculomas might be originated from the lung through such way.

The Symptom of intramedullary tuberculomas is similar with 
the clinical presentation of subacute spinal cord compression, such as motor and sensory symptoms, depending on the level of the lesion ${ }^{7,9,17,20)}$. There are incidences of weak lower extremity, back pain, bowel or bladder dysfunction, and paresthesia" that are similar with those in our case. In line with intracranial tuberculoma, it can be shown as an incidental finding or a symptomatic manifestation ${ }^{11,20)}$.

The diagnosis of the tuberculoma is made based on the neuroimaging findings, protein from purified derivative reactivity in cerebrospinal fluid, and histopathologic examination ${ }^{1)}$. The imaging modality of choice for diagnosis is MRI ${ }^{1,2)}$. MRI described three types of tuberculoma pictures depending upon the stage of tuberculoma, including noncaseating granuloma, caseating granuloma with a solid center, and caseating granuloma with liquid center"). In the early phase, the tuberculoma is characterized by severe infective reactions, poor formation of the gel capsule, and severe edema around the lesion. During this stage, tuberculomas appear isointense in T1 and T2 weighted images and enhanced after being intensified. As the gel content in the tuberculomas increases, the peripheral edema may decrease or disappear. As a result, T1 weighted images show isointense signal intensity. Meanwhile, T2 weighted images show isointense or low signal intensity. After the enhanced scanning, rim enhancement and a low signal in the central region were observed. With the development of caseation, T2 weighted images show a typical "target sign", which means that it exhibits a range from the low signal target to the high signal rim $^{1,6,11,14,20)}$. In the present case, MRI demonstrated images that are specific for noncaseating granuloma. In addition, helpful but nondiagnostic laboratory values include an elevated leukocyte count, an elevated sedimentation rate, and an elevated CSF protein with a predominance of mononuclear cells.

The ideal treatment of intramedullary tuberculoma remains controversial because there is no standardized treatment protocol caused by the rarity of this disease. Both surgical and medical treatments have yielded good results in different series $^{3,6,13,14,20)}$. Many authors have recommended to undergo a medical treatment first for the intramedullary tuberculomas with good results ${ }^{3,7,14,17)}$. Undertaking a surgery is generally indicated for cases with (1) uncertain diagnosis; (2) poor response to medical management; (3) progressive deterioration of neurologic status during medical treatment; and (4) progressive enlargement of the lesion that is observed on follow-up $\mathrm{MRI}^{3)}$. Our patient was diagnosed with paraplegia with classical imaging features of intramedullary tuberculoma and with documented pulmonary TB. Because of the neurologic deficit, surgical intervention was done. Our experience, although limited, has shown that neurological improvement is likely to occur when surgical intervention is undertaken before the damage in the irreversible cord takes place.

\section{CONCLUSION}

CNS TB causes significant morbidity and mortality. Moreover, diagnosis and treatment are difficult. Immunosuppression caused by immunosuppressive therapy after transplantation poses a number of difficulties such as significant increase in the risk of acquiring CNS TB, complicated clinical presentation, diagnosis, and treatment. In addition, it becomes a challenge for the clinician when he/she differentiates CNS TB from other potential opportunistic infections ${ }^{12)}$. Spinal intramedullary tuberculoma should be considered in differential diagnosis of the spinal cord lesion in patients with an indication of tuberculosis or an immunocompromised state. The combination of antituberculosis medication and microsurgical resection is considered as the optimal treatment. In particular, surgical decompression can be necessary in case of neurologic deficit.

\section{REFERENCES}

1. Bernaerts A, Vanhoenacker FM, Parizel PM, Van Goethem JW, Van Altena R, Laridon A, et al: Tuberculosis of the Central Nervous System: Overview of Neuroradiological Findings. Eur Radiol 13:1876-1890, 2003

2. Chitre PS, Tullu MS, Sawant HV, Ghildiyal RG: Co-Occurrence of Intracerebral Tuberculoma with Lumbar Intramedullary Tuberculoma. J Child Neurol 24:606-609, 2009

3. Devi BI, Chandra S, Mongia S, Chandramouli BA, Sastry KV, Shankar SK: Spinal Intramedullary Tuberculoma and Abscess: A Rare Cause of Paraparesis. Neurol India 50:494-496, 2002

4. Gökalp HZ, Ozkal E: Intradural Tuberculomas of the Spinal Cord. Report of Two Cases. J Neurosurg 55:289-292, 1981

5. Henderson C, Meyers B, Humayun Gultekin S, Liu B, Zhang DY: Intracranial Tuberculoma in a Liver Transplant Patient: First Reported Case and Review of the Literature. Am J Transplant 3:88-893, 2003

6. Jena A, Banerji AK, Tripathi RP, Gulati PK, Jain RK, Khushu $S$, et al: Demonstration of Intramedullary Tuberculomas by Magnetic Resonance Imaging: A Report of Two Cases. Br J Radiol 64:555-557, 1991

7. Lim YS, Kim SB, Kim MK, Lim YJ: Disseminated Tuberculosis of Central Nervous System: Spinal Intramedullary and Intracranial Tuberculomas. J Korean Neurosurg Soc 54:61-64, 2013

8. Lopez de Castilla D, Schluger NW: Tuberculosis Following Solid Organ Transplantation. Transpl Infect Dis 12:106-112, 2010

9. MacDonnell AH, Baird RW, Bronze MS: Intramedullary Tuberculomas of the Spinal Cord: Case Report and Review. Rev Infect Dis 12:432-439, 1990

10. Munoz P, Rodriguez C, Bouza E: Mycobacterium Tuberculosis Infection in Recipients of Solid Organ Transplants. Clin Infect Dis 40:581-587, 2005 
11. Muthukumar N, Venkatesh G, Senthilbabu S, Rajbaskar R: Surgery for Intramedullary Tuberculoma of the Spinal Cord: Report of 2 Cases. Surg Neurol 66:69-74, 2006.

12. Nelson CA, Zunt JR. Tuberculosis of the Central Nervous System in Immunocompromised Patients: Hiv Infection and Solid Organ Transplant Recipients. Clin Infect Dis 53:915-926, 2011

13. Nomura S, Akimura T, Kitahara T, Nogami K, Suzuki M: Surgery for Expansion of Spinal Tuberculoma During Antituberculous Chemotherapy: A Case Report. Pediatr Neurosurg 35:153-157, 2011

14. Rao GP: Spinal Intramedullary Tuberculous Lesion: Medical Management. Report of Four Cases. J Neurosurg 93:137-141, 2003

15. Singh N, Paterson DL: Mycobacterium Tuberculosis Infection in Solid-Organ Transplant Recipients: Impact and Implications for Management. Clin Infect Dis 27:1266-1277, 1998
16. Sohns JM, Staab W, Menke J, Bergau L, Dabir D, Schwarz A, et al: Vascular and Extravascular Findings on Magnetic Resonance Angiography of the Thoracic Aorta and the Origin of the Great Vessels. J Magn Reson Imaging 40:988-995, 2014

17. Thacker MM, Puri AI: Concurrent Intra-Medullary and IntraCranial Tuberculomas. J Postgrad Med 50:107-109, 2004

18. Torii H, Takahashi T, Shimizu H, Watanabe M, Tominaga T: Intramedullary Spinal Tuberculoma--Case Report. Neurol Med Chir (Tokyo) 44:266-268, 2004

19. Vishnubhotla S, Siddhartha B, Sivramakrishna G, Lakshmi AY, Kumaraswamy RM: Proptosis in a Post-Renal Transplant Patient with Disseminated Tuberculosis. Transpl Infect Dis 11:241-242, 2009

20. Yen HL, Lee RJ, Lin JW, Chen HJ: Multiple Tuberculomas in the Brain and Spinal Cord: A Case Report. Spine (Phila Pa 1976) 28:E499-502, 2003 